\title{
Successful treatment of granulomatous reactions secondary to injection of esthetic implants
}

Pedro Lloret, MD,* Agustín España, PhD, MD,* Ana Leache, MD,† Ana Bauzá, MD,* Marta Fernández-Galar, MD,* Miguel Angel Idoate, PhD, MD $\ddagger$ Gerd Plewig, PhD, MD,§ and Lothar Weber, PhD, MD\#

Departments of *Dermatology and $\ddagger$ Pathology, University Clinic of Navarra, Pamplona,Spain; †Clinica San Miguel, Pamplona, Spain; §Department of Dermatology, Ludwig Maximillian University, Munich, Germany; \#University of Ulm, Ulm, Germany

\section{BACKGROUND}

In recent years, various injectable materials have come into use to improve esthetic appearance.

\section{OBJECTIVE}

We describe the clinical and histopathologic aspects of two patients who received intradermal injections of an unknown dermal filler and the different diagnostic tools used to identify the unknown injected material (reflexion electron microscopy, electron dispersing $\mathrm{x}$-ray) and discuss the possibility of a metastatic granulomatous reaction in one patient. We also describe two treatments for this complication and evaluate the legal considerations of the use of materials that have been adulterated and/or whose composition is unknown to the patient.

\section{METHODS}

We present two patients who developed a granulomatous foreign-body reaction after the subcutaneous injection of an esthetic implant. We treated patient 1 with isotretinoin and 2 months later with doxycycline. We administered isotretinoin to patient 2 .

RESULTS. We observed a partial improvement in patient 1 after isotretinoin treatment and a remarkable improvement after administration of doxycycline. In patient 2, we observed an excellent response to isotretinoin.

\section{CONCLUSION}

Isotretinoin and doxycycline, when administered separately, seem to offer effective treatment for reactions resulting from silicone implants. However, further studies that include a larger number of patients and those with reactions secondary to other fillers are clearly needed before the effectiveness of this treatment can be confirmed.

Address correspondence and reprint requests to: Pedro Lloret, MD, Clinica Universitaria de Navarra, Dermatologia, Avda. Pio XII, 36, 31080 Pamplona (Navarra), España, or e-mail: pedrolloretluna@yahoo.com. 
Over the last few years, various materials have been used to improve esthetic appearance, including silicone, ethylmethacrylate, hyaluronic acid (eg, Dermalive), polymethylmethacrylate microspheres in partly denatured bovine collagen (eg, Artecoll), and others. These materials are usually well tolerated, but secondary local effects have been reported, mainly after using silicone. In addition, the possible induction of autoimmune diseases still remains controversial. We present two patients who developed a granulomatous foreign-body reaction after the subcutaneous injection of an esthetic implant in another medical center. We describe the clinical and histopathologic aspects and discuss the possibility of a metastatic granulomatous reaction in one patient, as well as the different diagnostic tools used to identify the unknown injected material. We evaluate the medical consequences of these injections, the different therapeutic approaches possible, and the legal considerations secondary to the use of materials that have been adulterated and/or whose composition is unknown to the patient.

\section{CASE REPORTS}

\section{Case 1}

A 68-year-old woman presented at our hospital with a 2-year history of perioral cutaneous induration and visible hypertrophy in this area. Approximately 5 years earlier, she had received subcutaneous injections of an unknown material in the labial folds in another hospital. In the last 2 years, the inflammation had been progressive, spreading to both cheeks and the perinasal area. The patient had been treated in other hospitals with deflazacort, methylprednisolone, betamethasone, and ceftriaxone, without any improvement. Clinical examination revealed violet patched perioral lesions with diffuse margins, pronounced hypertrophy, intense labial edema restricting mouth opening, and palpable skin induration in the perioral and cheek area (Figure 1A). A cutaneous biopsy specimen was obtained. Histologic examination showed a dense chronic inflammatory granulomatous infiltrate in the dermis and between striated muscle fibers, where multinucleated giant cells suggestive of foreign-body reaction were identified. Inside some giant cells, vacuoles were recognizable (Figure 1B), but no birefringent material was observed. The bacteriologic and mycologic cultures and ZiehlNeelsen staining from the skin specimen were negative. A reflexion electron microscopy (REM) probe demonstrated the presence of particles of material within the granuloma (Figure 1C), and electron dispersing x-ray (EDX) (Figure 1D) revealed high levels of silica, suggesting the existence of silicone. Cranial computed tomography was performed and revealed a diffuse reaction with vague margins, extending from the mandibular symphysis to the base of the nasal pyramid, involving the upper and lower lips (Figure 1E). Blood cell count and hepatic enzymes revealed no abnormal levels. The antinuclear antibody titer was 1/40. She was typed for HLA antigens and was found positive for A24, B44, CW4, DR4, DR52, DRB4, B35, BW4, BW6, and DR7. We started treatment with isotretinoin $(0.5 \mathrm{mg} / \mathrm{kg} / \mathrm{d}$ for 6 months $)$, combined with prednisone (20 mg for 1 week, and then the dose was gradually tapered), only for the first month of treatment. An improvement in inflammation, induration, and mouth opening was observed (Figure 1F). Two months after discontinuation of isotretinoin, doxycycline (100 mg/bid) was administered for 2 months, with further improvement. 


\section{Case 2}

A 65-year-old woman presented with a 2-year history of painful interciliary and supralabial induration. Two years before presentation, an unknown material had been injected subcutaneously at another medical center. She denied arthralgias, photosensitivity, or any other health problems. Intralesional corticosteroids had been infiltrated in another center, but a peripheral spread of the granuloma occurred. Physical examination revealed perioral edema and subcutaneous interciliary induration, $3 \mathrm{~cm}$ in diameter (Figure 2A), also in the left supraorbital area. An induration, $2.5 \mathrm{~cm}$ in width, was palpable close to the lower lip and chin and extended to both corners of the mouth. Histologic examination revealed granulomas and vacuoles inside giant cells (Figure 2B). REM showed particles of material within the granuloma (Figure 2C), and EDX analysis demonstrated the presence of silicone. The antinuclear antibody titer was 1/80. Expression of HLA antigens A11, B27, B35, BW4, BW6, DR7, DRW53, and DQ2 was found. Computed tomography demonstrated deposition of a subdermal substance without bone involvement (Figure 2D). After treatment with isotretinoin $(0.5 \mathrm{mg} / \mathrm{kg} / \mathrm{d}$ for 6 months), the indurated lesions disappeared (Figure 2E). Interestingly, although the patient developed no new facial lesions, 6 months after discontinuation of the treatment, some violet asymptomatic infiltrated lesions appeared on her lumbar area. Clinical examination revealed erythematoviolaceous plaques, ranging from 1 to $4 \mathrm{~cm}$ in diameter, on her lumbar area (Figure 2F) and enlargement of a laparotomy scar. Cell blood count and serum angiotensin-converting enzyme levels were in the normal range. Chest radiography revealed no alterations indicating pulmonary sarcoidosis. Histologic examination demonstrated a granulomatous foreign-body reaction, with vacuoles inside giant cells, compatible with distant spread of silicone (Figure 2G). Examination with EDX could not confirm the existence of silicone in this area.

\section{DISCUSSION}

Reports of granulomatous reactions secondary to substances used as esthetic implants are not infrequent. Sometimes, the patient is not informed about the material injected, and the identification and subsequent treatment are correspondingly difficult. Recently, Requena and colleagues established distinctive morphologic findings for each type of microimplant, ${ }^{1}$ which may be useful in distinguishing between the substances used. Thus, silicone granulomas show intracellular and extracellular nonbirefringent vacuoles, whereas Artecoll (polymethylmethacrylate microspheres in partly denatured 3.5\% bovine colla-gen) and Dermalive (hyaluronic acid with acrylic hydrogel) present only extracellular vacuoles. On the basis of these descriptions, the injected material in our patients may be silicone because we observed intracellular vacuoles in both biopsies. The histologic findings in our patients are characteristic of a foreign-body granulomatous reaction. However, histology is not accurate enough to identify these materials. REM and EDX seem to be useful diagnostic tools for identifying occult materials. ${ }^{2}$ These techniques point toward the presence of silicone in a cutaneous biopsy, as occurred in the probes we analyzed.

In our opinion, the correct management of these patients (Table 1) may consist of a physical examination and a laboratory analysis targeted to rule out an associated autoimmune disease. A skin biopsy must be performed, and it should be cultured and subjected to histologic examination and, if possible, to REM and EDX. Moreover, a radiographic study should be performed to exclude soft tissue and bone inflammation. 
The association between silicone and connective tissue diseases is controversial. The National Academy of Science (United States) ${ }^{3}$ and the Medical Device Agency (United Kingdom) concluded that there is not sufficient evidence to confirm this association. ${ }^{4}$ In the patients in our study, we found few clinical and laboratory data suggestive of an immunologic reaction. We observed low antinuclear antibody titers of $1 / 40$ and 1/80, respectively, and a granulomatous distant reaction in patient 2 . The pathophysiology of these reactions is controversial. The infiltrate of macrophages, lymphocytes, and giant cells has been suggested as occurring secondary to a cellular immunity phenomenon. ${ }^{5}$ An immune mechanism is also supposed in positive skin testings with hyaluronic acid performed in patients who developed skin reactions secondary to hyaluronic acid skin fillers. ${ }^{6}$ The observation of plasmatic cells suggests a humoral response, but no specific antibodies against silicone have been found. ${ }^{4,7}$ Silicone may also interact with serum proteins and act as an antigenic haptin stimulating inflammation. ${ }^{8,9}$ Moreover, HLA antigens may also play a role in granulomatous reactions. Thus, some HLA antigens have been found with an increased frequency in annular granuloma, sarcoidosis, and other granulomatous diseases. ${ }^{10,11}$ Interestingly, our two patients share common HLA antigens (B35, BW4, BW6, DR7). One of them, HLAB35, has also been described in annular granuloma, ${ }^{9}$ whereas HLA antigens DR5 and DR52, present in patient 1 , have also been observed in sarcoidosis. ${ }^{12}$

Several granulomatous reactions have been described after using medical and nonmedical-grade silicone. ${ }^{13-15}$ From the beginning of the medical use of this substance in 1940, local and distant complications have been observed, ${ }^{16,17}$ probably related to its ability to migrate through subcutaneous planes ${ }^{18}$ and to the lymph nodes. ${ }^{19}$ Although injected silicone was thought to be inert and safe, toxicity studies in animals have demonstrated that systemic administration of silicone is able to induce acute pulmonary edema, pneumonia, hepatic fibrosis, and death secondary to embolism. ${ }^{20-22}$ The injection of silicone in humans has been reported to induce local erythema, ecchymosis, ulceration, ${ }^{23}$ hyperpigmentation, or granulo-mas. Furthermore, even blindness has been described after injections close to the ophthalmic vessels. ${ }^{24}$ According to the literature, the incidence of granulomatous reactions is variable. Some authors have found these reactions in 13 of 92 patients injected, ${ }^{13}$ whereas others have reported no cases after 17,000 injections. ${ }^{25}$ Possible explanations for these disparities in incidence include the quantity of silicone injected, the presence of contaminants, and the depth of injection. Silicone implants have also been used in breast augmentation and have been found to cause arthritis, renal failure, scleroderma, and eosinophilic fasciitis, ${ }^{13,14,25-27}$ probably owing to an autoimmune reaction, although some recent reviews have questioned these findings. ${ }^{4}$ Recently, Suzuki and colleagues described a metastatic silicone granuloma and sicca complex in a silicone breast implant recipient. ${ }^{16}$ In our study, the presence of a granulomatous distant lesion with intracellular vacuoles in patient 2 points to the physical distant presence of silicone and not a systemic immune reaction to the esthetic implant. This distant presence is compatible with the ability of silicone to migrate through the lymph nodes or subcutaneous planes, as has been already described. ${ }^{18,19}$

Our patients did not respond to previous treatment with intralesional cortiicosteroids, which is one of the firstline treatment options (see Table 1). Other treatments that have been described include allopurinol, ${ }^{28}$ surgical excision, and immunomodulating agents (oral, intramuscular, intravenous, imiquimod). ${ }^{29}$ Jansen and colleagues described a patient with a granulomatous reaction to silicone that improved after the administration of isotretinoin, probably as a result of the anti-inflammatory effect described by Plewig and Wagner. ${ }^{2,30}$ In our study, we observed an evident improvement in patient 2 when treated with isotretinoin. Other authors have found that this drug is effective in other 
granulomatous diseases, such as actinic granuloma and granulomatous rosacea, ${ }^{11,31}$ in which exaggerated inflammation occurs. Furthermore, we observed a partial improvement in patient 1 after treatment with isotretinoin and a remarkable improvement after administration of doxycycline. We decided to administer doxycycline following the report of a clinical antiinflammatory response with tetracyclines in other granulomatous diseases, such as sarcoidosis. ${ }^{32}$

In view of the increasing use of esthetic implants, we will witness a rise in the incidence of reactions secondary to these injections. From a legal point of view, the injection of these materials must be approved (ie, in Spain, as in some other countries, the injection of silicone is forbidden), and the patient must provide written informed consent and must be informed of the exact composition and quantity of the material being injected. These data should be clearly noted in the patient's medical record.

\section{REFERENCES}

1. Requena C, Izquierdo MJ, Navarro M, et al. Adverse reactions to injectable aesthetic microimplants. Am J Dermatopathol 2001;23:197-202.

2. Jansen T, Kossmann E, Plewig G. Silikonome, ein interdisziplinäres Problem. Hautarzt 1993;44:636-43.

3. Tugwell P, Wells G, Peterson J, et al. Do silicone breast implants cause rheumatologic disorders? A systematic review for a court-appointed national science panel. Arthritis Rheum 2001;44:2477-84.

4. Perkins LL, Clark BD, Klein PJ, Cook RR. A meta-analysis of breast implants and connective tissue disease. Ann Plast Surg 1995;35:561-70.

5. Raszewski R, Guyuron B, Lash RH, et al. A severe fibrotic reaction after cosmetic liquid silicone injection: a case report. J Craniomaxillofac Surg 1990;18:225-8.

6. Lowe NJ, Maxwell CA, Lowe P, et al. Hyaluronic acid skin fillers: adverse reactions and skin testing. J Am Acad Dermatol 2001;45:930-3.

7. Nosanchuk JS. Injected dimethylpolysiloxane fluid: a study of antibody and histologic response. Plast Reconstr Surg 1968;42:562-6.

8. Heggers JO, Kossovsky N, Parsons RW, et al. Biocompatibility of silicone implants. Ann Plast Surg 1983;11:38-45.

9. Wedler FC. Analysis of biomaterials deposited on soft contact lenses. J Biomed Mater Res 1977;11:525.

10. Friedman SJ, Winkelmann RK. Familial granuloma annulare. Report of two cases and review of the literature. J Am Acad Dermatol 1987;16:600-5.

11. Ratnavel RC, Grant JW, Handfield-Jones SE, Norris PG. O’Brien's actinic granuloma: response to isotretinoin. J R Soc Med 1995;88:528P-9P.

12. Luisetti M, Beretta A, Casali L. Genetic aspects in sarcoidosis. Eur Respir 2000;16:768-80.

13. Wilkie TF. Late development of granuloma after liquid silicone injections. Plast Reconstr Surg 1977;60:179-88.

14. Spiera H. Scleroderma after silicona augmentation mammoplasty. JAMA 1988;260:236-8.

15. Rudolph R, Abraham J, Vecchione T, et al. Myofibroblasts and free silicone around breast implants. Plast Reconstr Surg 1978;62:185-95. 
16. Suzuki K, Aoki M, Kawana S, et al. Metastatic silicone granuloma: lupus miliaris disseminatus facei-like facial nodules and sicca complex in a silicone breast implant recipient. Arch Dermatol 2002;138:537-8.

17. Anderson DR, Schwartz J, Cottrill CM, et al. Silicone granuloma in acral skin in a patient with silicone-gel breast implants and systemic sclerosis. Int J Dermatol 1996;35:36-8.

18. Capozzi AR, Dubou R, Pennisi VR. Distant migration of silicone gel from a ruptured breast implant. Plast Reconstr Surg 1989;62:302-3.

19. Hausner RJ, Schoen FJ, Mendez-Fernandez MA, et al. Migration of silicone gel to axillary lymph nodes after prosthetic mammoplasty. Arch Pathol Lab Med 1981;105:371-2.

20. Celli B, Textor S, Kovnat D. Adult respiratory distress syndrome following mammary augmentation. Am J Med Sci 1978;275:81-5.

21. Chastre J, Basset F, Viau F, et al. Acute pneumonitis after subcutaneous injections of silicone in transsexual men. N Engl J Med 1983;308:764-7.

22. Kopf EH, Vinnik CA, Bongiovi JJ, Dombrowski DJ. Complications of silicone injections. Rocky Mt Med J 1976;73:77-80.

23. Rapaport MJ, Vinnik C, Zarem H. Injectable silicone: cause of facial nodules, cellulitis, ulceration, and migration. Aesthetic Plast Surg 1996;20:267-76.

24. Shin H, Lemke BN, Stevens TS, Lim MJ. Posterior ciliary-artery occlusion after subcutaneous silicone-oil injection. Ann Ophthalmol 1988;20:342-4.

25. Webster RC, Fuleihan NS, Hamdan US. Injectable silicone: report of 17,000 facial treatments since 1962. Am J Cosmetic Surg 1986;3:41-8.

26. Selmanowitz VJ, Orentreich N. Medical grade fluid silicone: a monographic review. J Dermatol Surg Oncol 1977;3:597-61.

27. Webster RC, Fuleihan NS, Gaunt JM. Injectable silicone for small augmentations: twenty year experience in humans. Am J Cosmetic Surg 1984;1:1-10.

28. Reisberg EM, Landthaler M, Wiest L, et al. Foreign body granulomas caused by polymethylmethacrylate microspheres: successful treatment with allopurinol. Arch Dermatol 2003;139:17-20.

29. Baumann LS, Halem ML. Lip silicone granulomatous foreign body reaction treated with Aldara (imiquimod 5\%). Dermatol Surg 2003;29:429-32.

30. Plewig G, Wagner A. Anti-inflammatory effects of 13-cis-retinoic acid: an in vivo study. Arch Dermatol Res 1981;270:89-94.

31. Smith KW. Perioral dermatitis with histopathologic features of granulomatous rosacea: successful treatment with isotretinoin. Cutis 1990;46:413-5.

32. Bachelez H, Senet P, Cadranel J, et al. The use of tetracyclines for the treatment of sarcoidosis. Arch Dermatol 2001;137:69-73. 

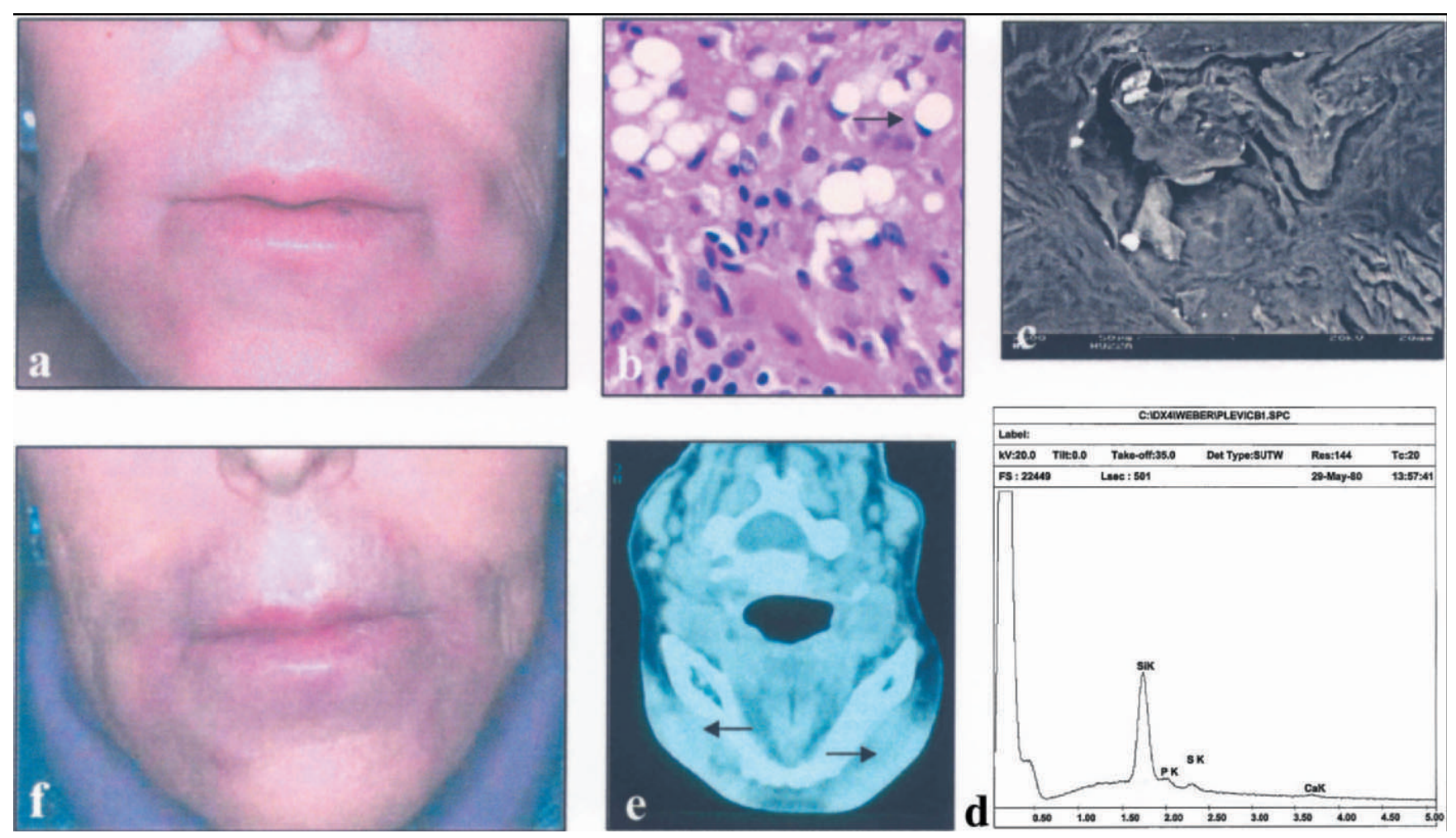

Figure 1. Patient 1: (A) Clinical picture before treatment: pronounced hypertrophy and intense labial edema; (B) histologic examination (hematoxylin-eosin stain; x400 original magnification): vacuoles inside giant cells; (C) reflexion electron microscopy: presence of particles of material within the granuloma; (D) electron dispersing $\mathrm{x}$-ray: high presence of silica, suggesting the presence of silicone; (E) cranial computed tomography: diffuse reaction from the mandibular symphysis to the base of the nasal pyramid; (F) clinical picture after treatment: improvement in inflammation, mouth opening, and induration. 

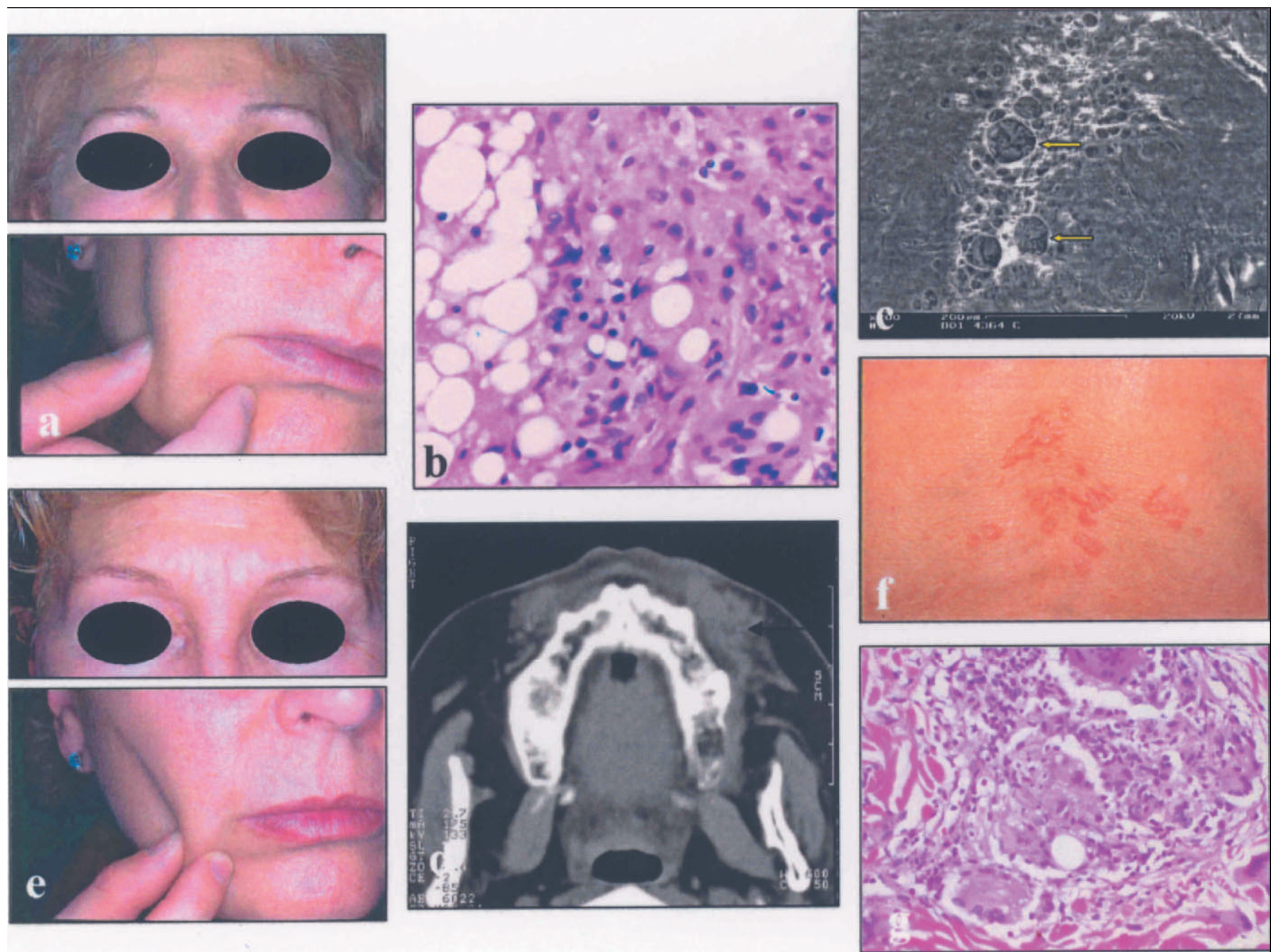

Figure 2. Patient 2: (a) clinical picture before treatment: interciliary, left supraorbital, lower lip, and chin subcutaneous induration; (b) histologic examination (hematoxylineosin stain; x 400 original magnification): granuloma and vacuoles inside giant cells; (c) reflexion electron microscopy: particles of material within the granuloma (arrows); (d) computed tomography: subdermal deposition without bone involvement; (e) clinical picture after treatment: indurated lesions have disappeared; (f) picture of the lumbar area (6 months after discontinuation of the treatment): violet asymptomatic infiltrated lesions; (g) histologic examination of lumbar lesions (hematoxylin-eosin stain; x 200 original magnification): granulomas with vacuoles inside giant cells. 
Table 1. Management of patients with adverse rReaction to esthetic implants (Presumably silicone)

History: injected substance, volume, evolution of the disease

Physical examination: rheumatoid manifestations

Analysis: ANA, ENA, ACE

Radiographic studies: CT and/or MRI

Histology: intra- and extracellular inclusions

Skin culture: fungal, mycobacteria

Determination of the injected material: electron dispersing $\mathrm{x}$-ray and reflexion electron microscopy

Treatment options:

- Local: corticosteroids: topical or intralesional

Topical imiquimod ${ }^{29}$

- Systemic: systemic corticosteroids

Systemic allopurinol ${ }^{28}$

Prednisone (0.3-05 mg/kg/d) 1 mo together with isotretinoin $(0.5 \mathrm{mg} / \mathrm{kg} / \mathrm{d} 5$ mo); if refractable: doxycycline $100 \mathrm{mg} / \mathrm{bid} 3 \mathrm{mo}$

Immunomodulating agents $^{29}$

- Surgery: surgical excision

ACE = angiotensin-converting enzyme; $\mathrm{ANA}=$ antinuclear antibodies; $\mathrm{CT}=$ computed tomography; ENA = antibodies to extractable nuclear antigens; MRI = magnetic resonance imaging. 